\title{
Topological magnetic textures in magnetic topological insulators
}

\author{
Nisarga Paul ${ }^{1,2}$ and Liang $\mathrm{Fu}^{2}$ \\ ${ }^{1}$ Department of Physics, Harvard University, Cambridge, Massachusetts, USA \\ ${ }^{2}$ Department of Physics, Massachusetts Institute of Technology, Cambridge, Massachusetts, USA
}

(Received 10 March 2021; accepted 7 July 2021; published 20 August 2021)

\begin{abstract}
The surfaces of intrinsic magnetic topological insulators (TIs) host magnetic moments exchange-coupled to Dirac electrons. We study the magnetic phases arising from tuning the electron density using variational and exact diagonalization approaches. In the dilute limit, we find that magnetic skyrmions are formed, which bind to electrons, leading to a skyrmion Wigner crystal phase while at higher densities spin spirals accompanied by chiral one-dimensional channels of electrons are formed. The binding of electrons to textures raises the possibility of manipulating textures with electrostatic gating. We determine the phase diagram capturing the competition of intrinsic spin-spin interactions and carrier density and comment on the possible application to experiments in magnetic TIs and spintronic devices such as skyrmion-based memory.
\end{abstract}

DOI: 10.1103/PhysRevResearch.3.033173

\section{INTRODUCTION}

Topological materials, topological excitations, and topological quantum effects have become a very active research frontier in condensed matter physics. Manifestations of topology in momentum space include the integer quantum Hall effect [1], Chern insulators [2], and topological insulators (TIs) $[3,4]$. In real space, topological order parameter configurations such as vortices, monopoles, and skyrmions are ubiquitous in spin systems and magnetism [5-11]. In particular, magnetic skyrmions have been the focus of much recent study, spurred by their observation in room-temperature materials [12-14], stability [15-17], and suitability in spintronics for next-generation memory devices [18].

Both momentum-space and real-space topology can play crucial roles in magnetic topological insulators, where Dirac electrons interact with topological spin textures associated with magnetic moments [19-21]. Recently, the stoichiometric compound $\mathrm{MnBi}_{2} \mathrm{Te}_{4}$, a TI containing a periodic sublattice of $\mathrm{Mn}^{2+}$ ions with magnetic moment $5 \mu_{B}$ (where $\mu_{B}$ is the Bohr magneton), was synthesized and studied for the first time $[22,23]$, opening the new field of intrinsically magnetic TIs. The compound comprises alternating quintuple layers of the topological insulator $\mathrm{Bi}_{2} \mathrm{Te}_{3}$ and ferromagnetic (FM) MnTe bilayers. A single septuple layer (SL) thin film exhibits ferromagnetic order. The exchange coupling between FM-ordered magnetic moments and Dirac surface electrons on the adjacent TI layer can open up a gap at the Dirac point and give rise to nontrivial topology in momentum space, characterized by quantized Chern number [24]. The resulting Chern insulator

Published by the American Physical Society under the terms of the Creative Commons Attribution 4.0 International license. Further distribution of this work must maintain attribution to the author(s) and the published article's title, journal citation, and DOI. and zero-field quantum anomalous Hall state have recently been observed in transport measurements on exfoliated fewlayer Mn-Bi-Te flakes.

Unlike TIs with randomly doped magnetic impurities, intrinsic magnetic TIs are stoichiometric compounds containing a lattice of magnetic atoms coupled to topological electrons. This feature not only promises a magnetic ordering and quantum anomalous Hall effect at temperatures as high as $50 \mathrm{~K}$ $[25,26]$ but also opens the exciting possibility of magnetic control of topological electronic properties and electrical control of magnetic order. In particular, magnetic textures such as spirals and skyrmions can arise at surfaces and interfaces from the chiral Dzyaloshinskii-Moriya (DM) interaction [27-29] due to broken inversion symmetry. Magnetic TI surfaces provide a platform of this kind. This motivates us to consider the effect of real-space magnetic structures on topological Dirac electrons, which may enable manipulating magnetic domains and textures by electric currents and electrostatic gating.

Motivated by recent advances in magnetic TIs and skyrmion physics, we study the magnetic phenomena arising from doping Dirac electrons. We focus separately on the cases of (a) a single added electron, (b) low carrier density, and (c) high carrier density. We predict the formation of skyrmion textures with localized Dirac modes for cases (a) and (b) and the formation of stripes with one-dimensional (1D) channels of chiral modes for case (c). Because the ground-state magnetic order parameter depends on carrier density, we obtain electrically tunable skyrmion and stripe phases whose periods vary with density.

As a potential application, we envision that intrinsic magnetic TIs can open the path for a memory device storing information using magnetic skyrmions in a low-carrierdensity material. The use of skyrmions for information storage has been reported in magnetic metals, where the exchange interaction couples itinerant electrons and localized spins. However, due to the large carrier density of metals, a large current density is required to drive skyrmions in writing and 
reading out data, which results in Joule heating and considerable power consumption. This drawback can be alleviated by working with skyrmions in a low-carrier-density, bulkinsulating material such as a magnetic TI. As we shall show, a skyrmion in a magnetic TI carries tightly bound electric charge. Under the right conditions, these charged skyrmions are the only charge carrier at low doping. Therefore a relatively small current is sufficient to drive the skyrmion motion.

Model. For the Hamiltonian of the 2D surface of a magnetic TI describing Dirac electrons coupled to a dense array of $N$ classical spins $\vec{S}=\left(S^{x}, S^{y}, S^{z}\right)$ we take

$$
H=H_{e}+H_{e S}+H_{S},
$$

where the first two terms,

$$
H_{e}=v_{F} \sum_{\mathbf{k}} \hat{c}_{\mathbf{k}}^{\dagger}\left(k_{x} \sigma^{y}-k_{y} \sigma^{x}-E_{F}\right) \hat{c}_{\mathbf{k}}
$$

and

$$
H_{e S}=-\frac{J}{\sqrt{N}} \sum_{\mathbf{k}, \mathbf{q}} \hat{c}_{\mathbf{k}+\mathbf{q}}^{\dagger} \vec{S}_{\mathbf{q}} \cdot \vec{\sigma} \hat{c}_{\mathbf{k}},
$$

are a Rashba Hamiltonian for massless Dirac electrons and an exchange interaction, respectively. The last term consists of intrinsic spin interactions, including a ferromagnetic exchange interaction, DM interaction, Zeeman field, and out-of-plane anisotropy, namely,

$$
\begin{aligned}
H_{S}= & -A \sum_{\mathbf{r}, i} \vec{S}_{\mathbf{r}} \cdot \vec{S}_{\mathbf{r}+\mathbf{e}_{i}}+D \sum_{\mathbf{r}, i}\left[\mathbf{e}_{i} \times\left(\vec{S}_{\mathbf{r}} \times \vec{S}_{\mathbf{r}+\mathbf{e}_{i}}\right)\right]_{z} \\
& -\vec{B} \cdot \sum_{\mathbf{r}} \vec{S}_{\mathbf{r}}-K \sum_{\mathbf{r}} S_{z \mathbf{r}}^{2}
\end{aligned}
$$

where $z$ is the out-of-plane direction. We have chosen a normalization so that $S_{\mathrm{r}}^{2}=1$. The form of the DM interaction above is appropriate for a lattice with $C_{n v}$ symmetry, favoring the formation of spirals rotating along the wave vector (Néel type), as opposed to orthogonal to the wave vector (Bloch type).

The phase diagram of the magnetic moments without coupling to electrons has been studied both theoretically $[17,30]$ and experimentally $[8,9,31,32]$. With vanishing Zeeman field and anisotropy, the ground state of $H_{S}$ is a periodically modulated spiral texture with wave vector $q \sim D / A$ and Néel wall-like rotation. With increasing anisotropy, the spiral degenerates into a system of domain walls which is energetically favorable over the uniform state when $\sqrt{D^{2} / A K}$ is above a threshold. Turning on an out-of-plane Zeeman field penalizes the large areas of antiparallel spins, resulting in a phase transition to a skyrmion crystal (SkX) above a critical field and to a uniform state above a higher critical field. As the temperature is increased the spiral or SkX order is destroyed.

We now consider the exchange coupling between localized spins and topological surface electrons. As we shall show below, the interplay between magnetic and electronic degrees of freedom leads to (1) a carrier-density-dependent magnetic phase diagram and (2) chiral electronic states. Furthermore, the exchange coupling is capable of driving the formation of charged skyrmions at small electron doping, and Coulomb repulsion is capable of leading to a skyrmion Wigner crystal (SWX).
The remainder of this paper is organized as follows. In Sec. II we study the formation of skyrmion textures from doping a single electron and the formation of a SWX for a very dilute density of electrons. In Sec. III we study the stripe phase which forms at finite density using exact diagonalization and discuss the 1D channels of chiral modes bound to the stripes. We then present a phase diagram of competing stripe orders when $H_{e S}$ and $H_{S}$ are both important, and in Sec. IV we conclude.

\section{SKYRMION FORMATION}

Equation (4) has a uniform ground state $\vec{S}_{\mathbf{r}}= \pm \hat{z}$ when the direct exchange interaction dominates, and the coupled electrons form two bands at energies $\pm \sqrt{J^{2}+v_{F}^{2}|\mathbf{k}|^{2}}$. At charge neutrality, the lower band is filled, and the addition of another electron incurs an energy cost of $2 J$ unless a nontrivial texture forms. Equation (4) is known to support skyrmions as excitations, so we investigate the electron spectrum in the presence of a skyrmion. We plot this spectrum in Fig. 1(a). We observe midgap bound states, indicating the possibility that the system will spontaneously form a skyrmion to accommodate the extra electron and a skyrmion Wigner crystal for a very dilute density of electrons [Fig. 1(b)].

Let us work in the strong- $J$ limit, neglecting energy costs associated with $H_{S}$. As a variational approach, we consider a $50 \times 50$ square lattice hosting a skyrmion texture and add a single electron to the charge-neutral state. Then we compare the total energy with that of the uniform state with a single added electron. We take a tight-binding approximation for $H_{e}$ suitable for the lattice, which we describe in Appendix B; it has a single Dirac cone at $\mathbf{k}=0$. We choose a momentum cutoff $|\mathbf{k}|<k_{\mathrm{UV}}$ to indicate the region of linear dispersion. We parametrize the skyrmion profiles, shown in Fig. 2, using the following ansatz:

$$
\begin{aligned}
\left(S^{x}, S^{y}, S^{z}\right) & =\frac{\left(w+\bar{w},-i(w-\bar{w}), 1-|w|^{2}\right)}{1+|w|^{2}}, \\
w(x, y) & =e^{i \gamma} \frac{b^{\alpha+\beta}}{\bar{z}^{\alpha} z^{\beta}}, \quad z=x+i y .
\end{aligned}
$$

This parametrization was chosen for its compact description of a four-parameter family of skyrmion profiles: $\gamma$ adjusts the in-plane spin orientation (we set $\gamma=\pi$ for a Néel-type skyrmion), $b$ sets the radius, $\alpha-\beta$ is the vorticity, and $\alpha+\beta$ defines the sharpness of the domain wall. For example, a hard-wall magnetic bubble of radius $b$ is recovered by taking $\alpha=\beta \rightarrow \infty$. Indeed, any smooth magnetic texture can be captured by some smooth function $w(x, y)$. Skyrmion textures resulting from holomorphic $w$, a special case, were discussed in Ref. [33], and a one-parameter family of skyrmions [34] is recovered by taking $\alpha=0, \beta=-1, \gamma=\pi / 2$. Higherwinding skyrmions, multiple skyrmions, or even a skyrmion crystal can be achieved by taking appropriate sums of the above ansatz. Such a sum may be preferable to the typical sinusoidal ansatz when the core size of the skyrmions is uncorrelated with their spacing, as in the skyrmion Wigner crystal we discuss shortly.

In Fig. 2 we show that the system prefers to form a Néel skyrmion (with vorticity 1) to accommodate the extra 
(a)
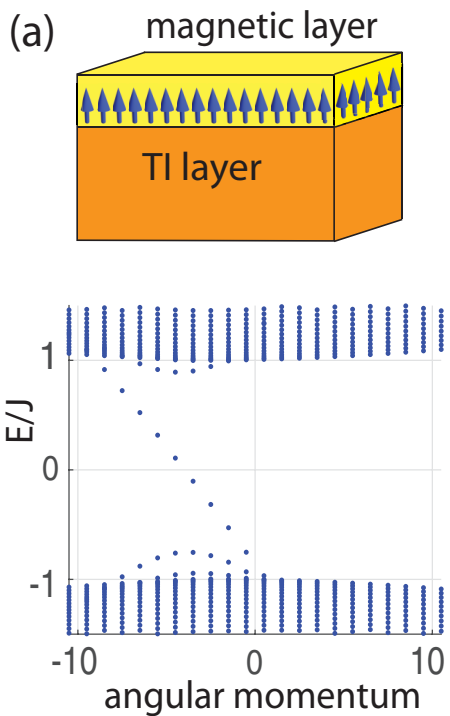

(b)
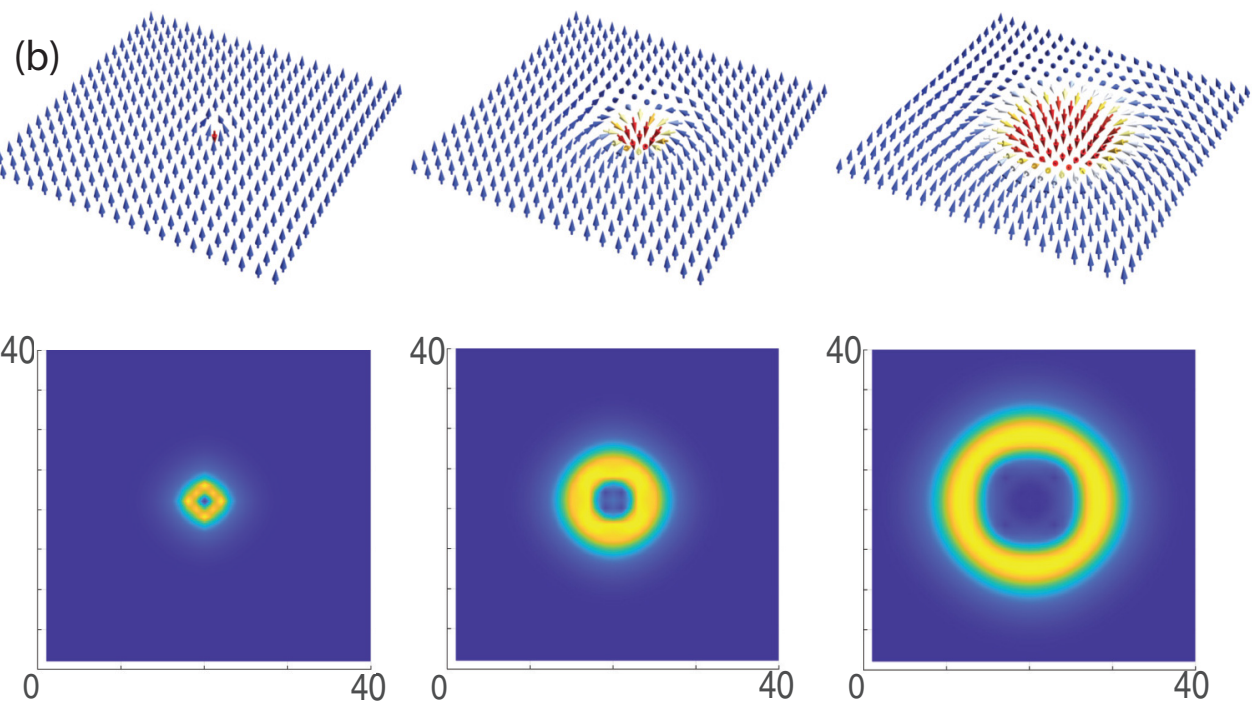

FIG. 1. (a) Top: Illustration of an intrinsic magnetic TI. In the case of $\mathrm{MnBi}_{2} \mathrm{Te}_{4}$ the alternating layers consist of the $\mathrm{TI} \mathrm{Bi}_{2} \mathrm{Te}_{3}$ and magnetic layer MnTe. The magnetic layer can host real-space topological textures such as Néel skyrmions, shown in (b). Bottom: Spectrum of Dirac electrons coupled to Néel skyrmion texture with radius $b=10$ lattice spacings and shape parameters $\alpha=3 / 2, \beta=1 / 2$ [Eq. (5)] plotted against total (spin + orbital) angular momentum, showing a branch of chiral midgap bound states. (b) Top: Néel skyrmion textures with radii $b=1,5$, and 10 lattice spacings and the same shape parameters. Bottom: Magnitude of spin-up component of the smallest $|E|$ Dirac bound state in the presence of the corresponding texture.

electron assuming a cutoff $k_{\mathrm{Uv}} a$ consistent with a Dirac cone dispersion, where $a$ is the lattice spacing. A Bloch skyrmion would be favored for a non-Rashba spin-orbit coupling. The added electron binds to the skyrmion with the profiles we numerically determined in Fig. 1. The radius $b$ of the skyrmion satisfies $a \lesssim b \lesssim 1 / k_{\mathrm{UV}}$. The cutoff dependence indicates that in real materials for which the Dirac cone is a low-energy approximation, the full dispersion may be relevant in determining skyrmion size. For instance, from angle-resolved photoemission spectroscopy (ARPES) data [22] for $\mathrm{MnBi}_{2} \mathrm{Te}_{4}$ the surface states' Dirac cone remains a good low-energy approximation up to $k_{\mathrm{UV}} \sim 0.05 \AA^{-1}$, beyond which the bulk bands are important. Since $a \approx 4 \AA$, our numerics would yield a skyrmion texture of $b / a \sim 3$, an extended object comprising $\sim 10 \mathrm{Mn}$ atoms. We note that although using the family of textures in Eq. (5) shows that in principle a skyrmion forms, we have not optimized over all skyrmion shapes. Moreover, for real materials the direct spin-spin interactions, including
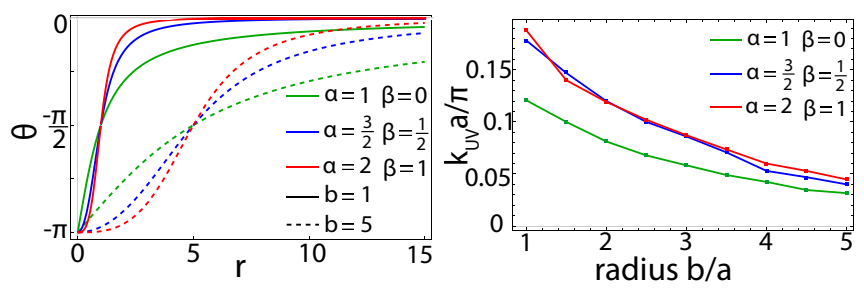

FIG. 2. Left: Skyrmion profiles $\theta(r)$, where $\theta=0$ corresponds to $+\hat{z}$, for the shape parameters shown [cf. Eq. (5)]. Right: The cutoff $k_{\mathrm{UV}}$ below which a skyrmion of the given radius becomes favorable upon doping a single electron. (Distances are measured in lattice spacings.) the ferromagnetic exchange and DM interaction, may also help to stabilize skyrmions and affect their size.

Let us approximate the spectrum of Dirac modes in the presence of a skyrmion. Working in polar coordinates, we consider the skyrmion of radius $b$

$$
\left(S^{r}, S^{\theta}, S^{z}\right)= \begin{cases}(0,0,-1) & r<b \\ (-1,0,0) & b<r<b+\delta \\ (0,0,1) & r>b+\delta\end{cases}
$$

with $\delta \ll b$. This is an idealized approximation to the skyrmions shown in Fig. 1. Dirac bound states are localized near $b$, with a half-integer angular momentum $m$ satisfying $|m| v_{F} / b \lesssim J$. We solve the continuum Dirac equation in detail in Appendix A. We find midgap energies

$$
E \approx m v_{F} / b-\delta J^{2} f\left(J b / v_{F}\right) / v_{F},
$$

where $f$ is a bounded, positive function of the skyrmion size whose explicit form is given in Eq. (A16). Thus a small inplane component $\delta>0$ results in a downward spectral shift, breaking particle-hole symmetry. This provides another way to understand why skyrmion formation is favorable.

The binding of Dirac modes to skyrmions was previously studied in Refs. [19,20]. Reference [19] studied the general relations between magnetic textures and their induced electric charge, as well as their motion in an electric field (see also Refs. [35,36]). Reference [20] studied the simplified case of a step-function out-of-plane skyrmion profile and found that the charged skyrmion is only energetically favorable with an external magnetic field due to particle-hole symmetry at zero field. However, realistic skyrmion profiles are smooth and contain an in-plane region, which breaks particle-hole symmetry. Our results show that for realistic profiles with broken 
particle-hole symmetry, charged skyrmions may be favorable even at zero external field.

The binding of charges to skyrmions is also seen in the quantum Hall ferromagnet, for instance, at the filling factor $v=1$. In this state, the Coulomb repulsion favors the spontaneous polarization of electron spins, and doping the $v=1$ ferromagnet leads to electrically charged skyrmion textures [37-39]. The quantum anomalous Hall state in intrinsic magnetic topological insulators differs from the quantum Hall ferromagnet in several key aspects. First, its ferromagnetism comes from the ordering of local magnetic moments (with $\sim 5 \mu_{B}$ per $\mathrm{Mn}$ atom in the case of $\mathrm{MnBi}_{2} \mathrm{Te}_{4}$ ), rather than the spins of low-density charge carriers. Second, spin-momentum locking in the Dirac surface states of a magnetic topological insulator leads to a strongly anisotropic magnetic response to the exchange field: An out-of-plane field opens a gap at charge neutrality, while an in-plane field does not. Therefore the Chern-Simons effective theory for quantum Hall ferromagnets cannot be applied to describe the skyrmion physics in magnetic topological insulators.

Very dilute limit. Since the large-S local magnetic moments are treated as classical, the skyrmions and Dirac electrons bound to them are immobile. When the density of added electrons is sufficiently low, the Coulomb repulsion is expected to drive a Wigner crystal phase of charged skyrmions. There are two distinct length scales in this phase: the lattice constants of the SWX, set by the density of doped electrons, and the skyrmion size, set by the exchange coupling and the spin susceptibility of Dirac electrons. The first length scale can far exceed the second. We emphasize that the SWX proposed here is driven by Wigner crystallization of charged skyrmions. This mechanism is similar to the SWX phase observed in the lightly doped $v=1$ quantum Hall ferromagnet [40-42], but different from the skyrmion crystal phase in helimagnets that is driven by DM interaction.

\section{STRIPE PHASES}

In this section we consider a finite electron density above charge neutrality. We work with the same tight-binding model (Appendix B) and the strong- $J$ limit so that electrons actively dictate the ground state. We find that the ferromagnetic state is unstable to a state with spatially modulated magnetization above a critical electron density, as shown in Fig. 3(a). Above the transition the preferred wave vector is $\sim 2 k_{F}$.

Let us comment on the band structure of Dirac electrons in the presence of a spiral phase. We expect a network of 1D modes localized along the regions where spins are in plane [43], as in Fig. 3(c). To see this, let us focus on a single such region, which we take along the $y$ axis, and approximate the spin texture in the vicinity as

$$
\vec{S}(x, y)=(1,0, q x) .
$$

The normalization $S^{2}=1$ is maintained to linear order in $x$. Solving the corresponding continuum equation

$$
\left[v_{F}\left(-i \partial_{x} \sigma^{y}-k_{y} \sigma^{x}\right)-J\left(\sigma^{x}+q x \sigma^{z}\right)\right] \psi=E \psi,
$$

we obtain the bound state

$$
\psi_{k_{y}}(x, y)=C e^{i k_{y} y} e^{-\frac{J q x^{2}}{2 v_{F}}}(1,-1)^{T}
$$

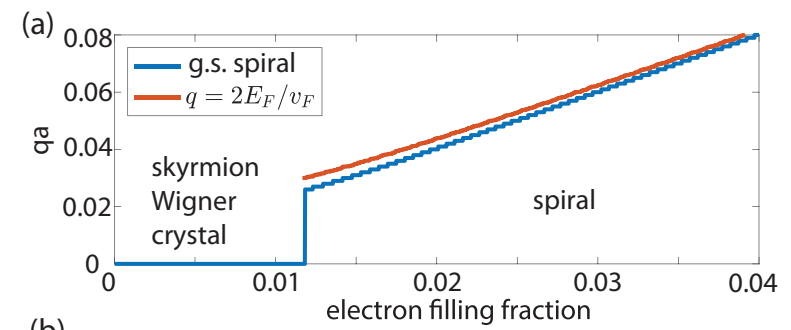

(b)

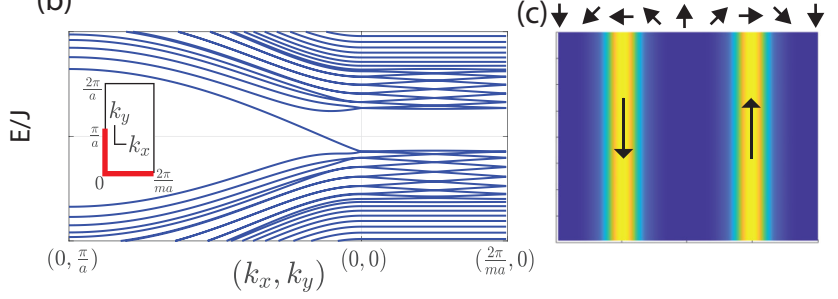

FIG. 3. (a) Ground-state (g.s.) sinusoidal Néel spiral of $H_{e}+$ $H_{e S}$, characterized by wave vector $q$ in the $x$ direction, as a function of doping. The slope $\sim 2 k_{F}$ for the underlying dispersion. A $10^{3} \times 500$ lattice with unit spacing and cutoff $k_{\mathrm{UV}}=\pi / 20 a$ was used. (b) Spectrum along the red line of the inset mini Brillouin zone (BZ) for Dirac electrons in a sinusoidal Néel spiral of wavelength $m a$ in the $x$ direction (here, $m=20, J=0.5$ ). Conduction band states descend to form midgap bound states localized in the $x$ direction. Localization leads to nearly flat bands in the $k_{x}$ direction, with dispersion tuned by $J$. (c) Magnitude of spin-up component of $E=0$ Dirac bound states in the presence of the spin spiral above. The velocities are indicated by long arrows.

with linear dispersion $E=-\operatorname{sgn}(q)\left(v_{F} k_{y}+J\right)$. This state is Gaussian localized in the $x$ direction over a length $\sim \sqrt{v_{F} / J q}$. It follows that in the presence of a spiral texture $\vec{S}=$ ( $\cos q x, 0, \sin q x)$, well-localized, approximately degenerate chiral modes form when $J / v_{F} \gg q$, in which case Eq. (8) is a valid approximation.

In Fig. 3(b) we plot the full spectrum of Dirac electrons in a Néel spiral texture to show that the linear branch of chiral modes descends into the gap from the conduction band, giving another picture for why a spiral state becomes favorable. Moreover, a spiral state in the appropriate limit would exhibit highly anisotropic conductance; the conductance would be much greater in the $y$ direction due to the $1 \mathrm{D}$ channels of bound states. Examples of such bound states are shown in Fig. 3(c).

Since the exchange coupling $J$ is small compared with the bandwidth of TI surface states (on the order of eV), the leading-order effect of $J$ on the spin degrees of freedom is to modify the spin-spin interaction through the Ruderman-Kittel-Kasuya-Yosida (RKKY) mechanism. The effective Hamiltonian for the array of spins, after integrating out the electrons, is given by

$$
H_{\mathrm{eff}}=-\sum_{\mathbf{q} a b} \chi_{\mathrm{tot}}^{a b}(\mathbf{q}) S_{-\mathbf{q}}^{a} S_{\mathbf{q}}^{b},
$$

where

$$
\chi_{\text {tot }}^{a b}(\mathbf{q})=J^{2} \chi_{e S}^{a b}(\mathbf{q})+\chi_{S}^{a b}(\mathbf{q}) .
$$

The spin susceptibility $\chi_{\text {tot }}(\mathbf{q})$ is a $3 \times 3$ Hermitian matrix whose largest eigenvalue and corresponding eigenvector 


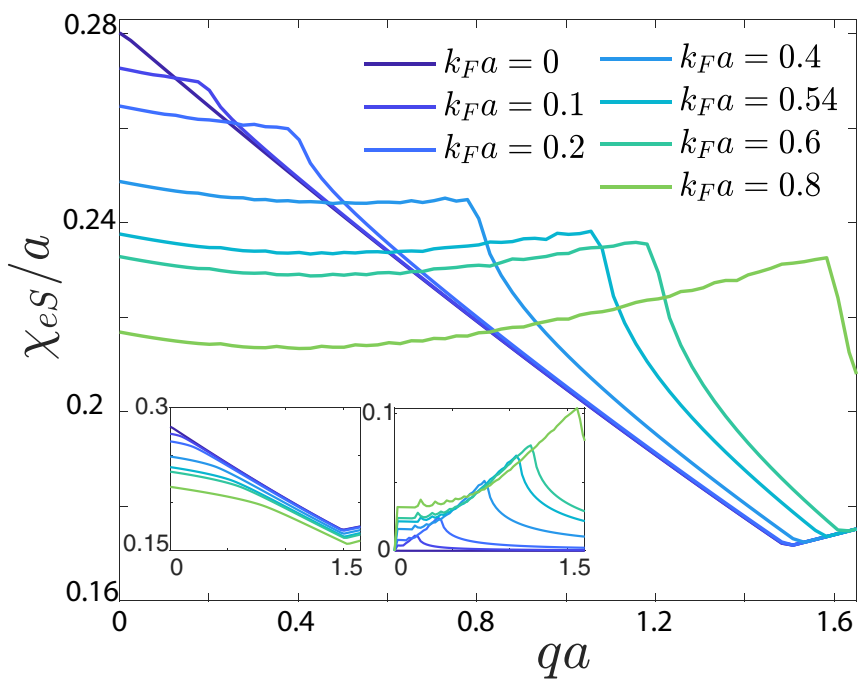

FIG. 4. The largest eigenvalue $\chi_{e s}(q)$ of the spin susceptibility defined in Eq. (11). The dominant peak shifts discontinuously away from $q=0$ at a critical $k_{F} a \approx 0.54$. The transition occurs at a point in the Brillouin zone where a Dirac cone is a good approximation to the dispersion of the $10^{3} \times 500$ lattice model $(a=1)$. Left and right insets display the inter- and intraband spin susceptibilities, respectively. The $2 k_{F}$ peak is entirely due to the latter, and the UV dependence is captured entirely in the former.

describe the wave vector and polarization of the ground-state spin texture. The first contribution is derived from secondorder perturbation theory in $J a / v_{F}$, while the second term can be read off from $H_{S}$ when the Zeeman field is absent. Their explicit forms are given in Eqs. (13) and (18). In the strong- $J$ limit we drop $\chi_{S}$ and focus only on $\chi_{e S}$. The latter can can be written as $U^{\dagger} \tilde{\chi} U$ with $U$ being a $3 \times 3$ unitary effecting a $\pi / 2$ rotation about $\hat{z}$ and

$$
\tilde{\chi}^{a b}(\mathbf{q})=\frac{-1}{2 N} \sum_{\mathbf{k}, s_{1}, s_{2}} \frac{f_{\mathbf{k}, s_{1}}-f_{\mathbf{k}+\mathbf{q}, s_{2}}}{\xi_{\mathbf{k} s_{1}}-\xi_{\mathbf{k}+\mathbf{q}, s_{2}}} F_{\mathbf{k} s_{1} ; \mathbf{k}+\mathbf{q}, s_{2}}^{a} F_{\mathbf{k}+\mathbf{q}, s_{2} ; \mathbf{k} s_{1}}^{b}
$$

with $\xi_{\mathbf{k} s}=s\left|v_{F}\right| k-E_{F}$ and $f_{\mathbf{k} s}=\left[1+e^{\beta \xi_{\mathbf{k} s}}\right]^{-1}$ being the Fermi distribution [44]. The $F^{a}$ are given by

$$
\begin{gathered}
F_{\mathbf{k}_{1} s_{1} ; \mathbf{k}_{2} s_{2}}^{x}=\frac{\operatorname{sgn}\left(v_{F}\right)}{2}\left(s_{1} e^{i \theta_{\mathbf{k}_{1}}}+s_{2} e^{-i \theta_{\mathbf{k}_{2}}}\right), \\
F_{\mathbf{k}_{1} s_{1} ; \mathbf{k}_{2} s_{2}}^{y}=-i \frac{\operatorname{sgn}\left(v_{F}\right)}{2}\left(s_{1} e^{i \theta_{\mathbf{k}_{1}}}-s_{2} e^{-i \theta_{\mathbf{k}_{2}}}\right), \\
F_{\mathbf{k}_{1} s_{1} ; \mathbf{k}_{2} s_{2}}^{z}=-\frac{1}{2}\left(1-s_{1} s_{2} e^{i\left(\theta_{\mathbf{k}_{1}}-\theta_{\mathbf{k}_{2}}\right)}\right),
\end{gathered}
$$

where $e^{i \theta_{\mathbf{k}}}=\left(k_{x}+i k_{y}\right) / k$

In Fig. 4 we plot the dominant eigenvalue $\chi_{e S}(q)$ of the spin susceptibility varying the electron doping $k_{F}$ (taking $\left.v_{F}=1\right)$. A square lattice with lattice constant $a$ is used as a UV completion. The plot has a peak at $q=0$ for small doping which trades dominance with a peak at $q=2 k_{F}$ at some critical $k_{F}$. This corresponds to the transition from a uniform state to a $2 k_{F}$ spiral, in accord with Fig. 3(a). The corresponding eigenvector describes a spiral texture with Néel wall-like rotation. For a non-Rashba spin-orbit coupling a Bloch wall-like rotation is favored. Figure 4 is also consistent with a skyrmion state comprising a sum of multiple magnitude- $2 k_{F}$ wave vectors, because $\chi_{e s}$ is radially symmetric up to isotropybreaking terms originating from the underlying lattice.

We refer to Eq. (13) with $s_{1}=s_{2}$ as the intraband susceptibility and with $s_{1}=-s_{2}$ as the interband susceptibility. The interband susceptibility is a UV divergent contribution (cut off by the lattice spacing) which occurs even when the chemical potential is zero and depends weakly on $q$. The intraband susceptibility is independent of the UV cutoff and dominated by Fermi surface contributions. These are both plotted in Fig. 4 (insets).

To see how the SWX phase fits into the picture presented in this section, we remark that the presence of two length scales in the SWX phase at low doping leads to multiple peaks in the Fourier transform of its magnetic structure, and the largest peak is at wave vector $\mathbf{q}=0$. Thus the closest singlewave-vector approximation is the uniform state, consistent with Figs. 3(a) and 4 in the low-density regime. Higher-order effects in the perturbative expansion in the exchange coupling $J$, higher harmonics in $\mathbf{q}$, and the inclusion of Coulomb repulsion are needed to fully capture the SWX phase.

Intermediate regime. We have previously worked at strong coupling, neglecting the effects of intrinsic magnetism. Now we allow the couplings of $H_{S}$ and $H_{e S}$ to be of the same order. The dominant eigenvalue of the total spin susceptibility [Eq. (12)] determines the ground-state properties of the system, where $\chi_{e S}$ was discussed in the previous section and $\chi_{S}$ can be read off from $H_{S}$ in the case of zero Zeeman field. Since a square lattice was used for $\chi_{e s}$, we use the same for $\chi_{S}$, although the only UV sensitivity will come from the interband susceptibility of $\chi_{e s}$, which affects the transition from $q=0$ to $q=2 k_{F}$. Thus we have

$$
\left.H_{S}\right|_{B=0}=-\sum_{a b} \chi_{S}^{a b}(\mathbf{q}) S_{-\mathbf{q}}^{a} S_{\mathbf{q}}^{b}
$$

with $\chi_{S}(\mathbf{q})$ given by

$$
\begin{aligned}
& \chi_{S}^{x x}=\chi_{S}^{y y}=A\left(\cos q_{x} a+\cos q_{y} a\right), \\
& \chi_{S}^{z z}=A\left(\cos q_{x} a+\cos q_{y} a\right)+K, \\
& \chi_{S}^{x z}=\left(\chi_{S}^{z x}\right)^{*}=i D \sin q_{x} a, \\
& \chi_{S}^{y z}=\left(\chi_{S}^{z y}\right)^{*}=i D \sin q_{y} a, \\
& \chi_{S}^{x y}=\chi_{S}^{y x}=0 .
\end{aligned}
$$

In terms of a rescaled coupling

$$
d^{2}=2 D^{2} / A K
$$

the dominant eigenvalue of $\chi_{S}$ is peaked at $q=0$ for $d<1$ and at

$$
q_{0}=\tan ^{-1}\left(\sqrt{d^{4}-1} / \sqrt{2 d^{2} A / K+1}\right) / a
$$

for $d \geqslant 1$ if $\mathbf{q}$ is taken along an axis of symmetry. The ground state of the system is a spin texture with wave vector $q=0,2 k_{F}$, or $q_{0}$, determined by the peak of the dominant eigenvalue of $\chi_{\text {tot }}$. This competition yields the phase diagram shown in Fig. 5, plotted in the $\left(d, k_{F}\right)$ plane with $A=K=$ $0.1, J=0.5$.

This phase diagram exhibits several interesting features. Increased electron doping expands the $q=q_{0}$ phase, thereby acting as an "effective DM interaction," an effect noted in 


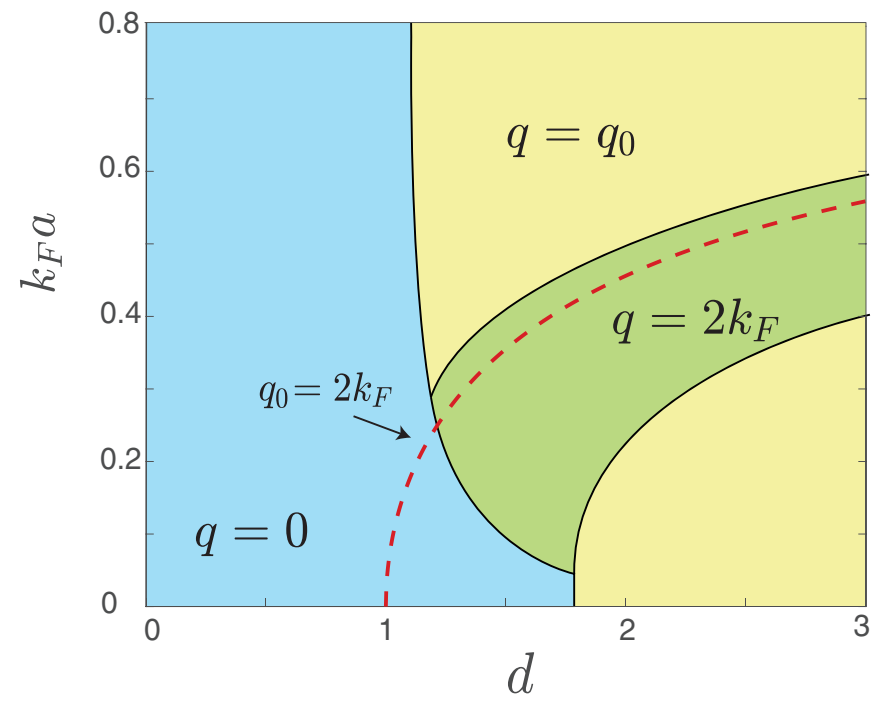

FIG. 5. Phase diagram describing Dirac electrons coupled to spins as the electron density and DM interaction are varied [within $\left.\left(\Delta d, \Delta k_{F} a\right)=(0.15,0.04)\right]$ with $A=K=0.1, J=0.5$, and $a=1$ on a $100 \times 100$ lattice. The spins exhibit stripe orders at wave vectors $q=0,2 k_{F}$, and $q_{0}$ [defined in Eq. (20)], as determined by the spin susceptibility peak.

recent works $[45,46]$. The line $q_{0}=2 k_{F}$ broadens into the RKKY phase with increasing exchange coupling $J$. When $J$ vanishes, this phase vanishes, and the $q=0$ and $q_{0}$ phases are separated by the transition at $d=1$. In the opposite limit $J \gg A, K$, the $q=q_{0}$ phase vanishes, and we recover the transition in Fig. 4 as a function of $k_{F}$. We note that for weak exchange coupling $J$, one expects these phases to be stripe orders (single $\mathbf{q}$ ) rather than multiple $\mathbf{q}$ because for $J=0$ the stripe phase is known to be the ground state at zero external field.

\section{DISCUSSION}

We have investigated the phases of an array of magnetic moments coupled to Dirac electrons upon tuning the electron density, motivated by intrinsic magnetic TIs subject to electrostatic gating.

We found that at very dilute densities, electrons bind to magnetic skyrmions, and we conjecture that an SWX results; at higher densities the SWX gives way to spin spirals bound to chiral electron channels. The DM interaction resulting from broken inversion symmetry is essential for the formation of skyrmions and stripes. Tuning the DM interaction and electron density reveals a phase diagram of stripe orders captured in Fig. 5. The interplay of real-space magnetic structures and topological Dirac electrons suggests the manipulation of magnetic domains and textures by electric currents, and electrostatic gating is possible with low dissipation, an attractive prospect for skyrmion-based information devices.

An interesting future direction is the study of the effects of skyrmion and spiral phases on transport phenomena and quantum oscillations. In a skyrmion crystal phase the Berry flux attached to each skyrmion should lead to topological density-of-states (DOS) oscillations which impact all physical observables [47]. The emergent orbital magnetic field of the nontrivial spin textures leads to a topological contribution in the Hall resistivity $\rho_{x y}(B)$, observed in $\mathrm{Mn}_{2} \mathrm{CoAl}$ thin films [48], Mn-doped $\mathrm{Bi}_{2} \mathrm{Te}_{3}$ quintuple layers [49], and correlated oxide thin films $[50,51]$ at low temperatures. However, anomalous features in Hall resistivity could originate from surface and bulk ferromagnetism instead of skyrmion physics [52]. We leave the precise form of the contributions from topological magnetism to physical observables to future work. We hope that the magnetic phase transitions predicted in this paper may spark further interest in magnetic topological insulators.

Note added. Recently, two related works on spin textures in magnetic topological insulators have appeared $[53,54]$.

\section{ACKNOWLEDGMENTS}

We thank Sungjoon Hong, Noah Yuan, and Hiroki Isobe for participation in and contributions to the early phase of this project. This work is supported by DOE Office of Basic Energy Sciences, Division of Materials Sciences and Engineering, under Award No. DE-SC0018945. L.F. is partly supported by a Simons Investigator award from the Simons Foundation.

\section{APPENDIX A: DIRAC ELECTRONS BOUND TO A SKYRMION}

Here, we find the bound-state spectrum of Dirac electrons in the presence of a skyrmion spin texture. The spin texture is defined on a lattice, but it is convenient to take a continuum approximation

$$
H_{e}=\int \psi^{\dagger} \mathcal{H} \psi, \quad \mathcal{H}=v_{F}\left(k_{x} \sigma^{y}-k_{y} \sigma^{x}\right)-J \vec{S} \cdot \vec{\sigma} .
$$

First we consider an idealized skyrmion of radius $b$, with

$$
S^{z}(r)= \begin{cases}-1 & r<b \\ +1 & r>b\end{cases}
$$

and $S^{x}=S^{y}=0$. In radial coordinates and setting $v_{F}=J=$ 1 , we may write

$$
\mathcal{H}=\sigma^{\theta}\left(-i \partial_{r}\right)+\frac{1}{r} \sigma^{r}\left(-i \partial_{\theta}\right)-S^{z} \sigma^{z} .
$$

It is convenient to define $\Lambda=\sqrt{r} e^{-\frac{i}{2} \sigma^{z}\left(\theta+\frac{\pi}{2}\right)}$, which satisfies

$$
\Lambda^{-1}\left(\sigma^{r}, \sigma^{\theta}, \sigma^{z}\right) \Lambda=\left(-\sigma^{y}, \sigma^{x}, \sigma^{z}\right),
$$

and consider the isospectral Hamiltonian

$$
\mathcal{H}^{\prime}=\Lambda^{-1} \mathcal{H} \Lambda=\sigma^{x}\left(-i \partial_{r}\right)-\frac{1}{r} \sigma^{y}\left(-i \partial_{\theta}\right)-S^{z} \sigma^{z} .
$$

Since $\mathcal{H}^{\prime}$ has no explicit $\theta$ dependence, we replace $\left(-i \partial_{\theta}\right)$ with a half-integer angular momentum $m$. Note that $m$ is a halfinteger because $\Lambda$ changes the azimuthal boundary conditions from periodic to antiperiodic. We would like to solve

$$
\left[\sigma^{x}\left(-i \partial_{r}\right)-\frac{m}{r} \sigma^{y}-S^{z}(r) \sigma^{z}\right]\left(\begin{array}{l}
f_{1} \\
f_{2}
\end{array}\right)=E\left(\begin{array}{l}
f_{1} \\
f_{2}
\end{array}\right) .
$$


Acting with $\left(-i \partial_{r} \pm i \frac{m}{r}\right)$ on the top and bottom row, we get

$$
\begin{aligned}
& -f_{2}^{\prime \prime}+\frac{m(m-1)}{r^{2}} f_{2}=\left(E^{2}-1\right) f_{2}, \\
& -f_{1}^{\prime \prime}+\frac{m(m+1)}{r^{2}} f_{1}=\left(E^{2}-1\right) f_{1},
\end{aligned}
$$

respectively, away from $r=b$. These can be solved as

$$
\begin{gathered}
f_{2}(x)=\sqrt{x}\left[\alpha J_{m-\frac{1}{2}}(-i x)+\beta Y_{m-\frac{1}{2}}(-i x)\right], \\
f_{1}(x)=-s \sqrt{x} \sqrt{\frac{1+s E}{1-s E}}\left[\alpha J_{m+\frac{1}{2}}(-i x)+\beta Y_{m+\frac{1}{2}}(-i x)\right]
\end{gathered}
$$

with $x=r \sqrt{1-E^{2}}, S^{z}(r)=s= \pm 1$, and $\alpha, \beta$ undetermined. There are several constraints on physical solutions, such as convergence of $\int d \theta d x x^{2}|f|^{2}$ and conservation of probability current. Solutions in $r>b$ are restricted by convergence to have $\beta=-i \alpha$ and take the form

$$
\begin{gathered}
f_{2}(x)=\alpha^{\prime} i^{m+\frac{1}{2}} \sqrt{x} K_{m-\frac{1}{2}}(x), \\
f_{1}(x)=\alpha^{\prime} i^{m-\frac{1}{2}} \sqrt{\frac{1+E}{1-E}} \sqrt{x} K_{m+\frac{1}{2}}(x) .
\end{gathered}
$$

Solutions in $r<b$ are restricted by conservation of probability current at the origin (all $\mathrm{m}$ ) or convergence (for $|\mathrm{m}|>$ $1 / 2$ ) to have $\beta=0$ and take the form

$$
\begin{gathered}
f_{2}(x)=\alpha^{\prime \prime} \sqrt{x}(-i)^{m-\frac{1}{2}} I_{m-\frac{1}{2}}(x), \\
f_{1}(x)=\alpha^{\prime \prime} \sqrt{\frac{1-E}{1+E}} \sqrt{x}(-i)^{m+\frac{1}{2}} I_{m+\frac{1}{2}}(x)
\end{gathered}
$$

using $J_{v}(-i x)=(-i)^{v} I_{\nu}(x)$. Continuity at $x_{0}=b \sqrt{1-E^{2}}$ fixes $\alpha^{\prime}$ and the energy:

$$
1=\frac{1+E}{1-E} \frac{I_{m+\frac{1}{2}}\left(x_{0}\right) K_{m-\frac{1}{2}}\left(x_{0}\right)}{I_{m-\frac{1}{2}}\left(x_{0}\right) K_{m+\frac{1}{2}}\left(x_{0}\right)} .
$$

This is a transcendental equation we can solve to get the bound-state spectrum for any angular momentum $m$ and radius $b$. The bound states have $E \approx m / b$ with great accuracy for large $b$ and some deviation at small $b$. They exist only for $|m| \lesssim b$. Importantly, they are localized near the radial domain wall $r=b$.

Indeed, one could have approximated this spectrum to great accuracy with the ansatz of a -1 eigenspinor of $\sigma^{y}$. Then one finds $f_{1}, f_{2} \sim \exp \left[-S^{z}(r) r\right]$ from Eq. (A6), implying that $f_{1}, f_{2}$ are exponentially localized near $b$. This allows the approximation $m / r \approx m / b$ to reproduce the bound-state spectrum.

This approximation is useful when we consider what happens upon introducing a small radial in-plane region to the spin texture, i.e., a region $(b, b+\delta)$ in which $S^{z}=0$ and $S^{r}=$ \pm 1 . Keeping in mind our choice of $\Lambda$ in Eq. (A4), we observe that this is captured by the perturbation $\theta(r-b) \theta(b+\delta-$ $r)\left(\mp \sigma^{y}+\sigma^{z}\right)$. Since $\left(f_{1}, f_{2}\right)^{T}$ is an approximate -1 eigenspinor of $\sigma^{y}$, we find a uniform $m$-independent spectral shift in first-order perturbation theory, breaking particle-hole symmetry. To leading order in $\delta$,

$$
\Delta E \approx \mp \delta \frac{4 b^{2} e^{-2 b}}{\left(4 b^{2}+2\right) \cosh 2 b-4 b \sinh 2 b-1} .
$$

We confirmed the shift by full analytical solution.

For comparison, we investigate the bound states of Schrödinger electrons in the same idealized skyrmion texture, Eq. (A2), with Hamiltonian

$$
\mathcal{H}=\frac{1}{2 \mu}\left(k_{x}^{2}+k_{y}^{2}\right) \sigma^{z}-J \vec{S} \cdot \vec{\sigma} .
$$

In radial coordinates, replacing $-i \partial_{\theta}$ with angular momentum $m$ and taking an $s$ eigenstate of $\sigma^{3}$, the Schrödinger equation becomes

$$
\frac{1}{2 \mu}\left[-\partial_{r}^{2}-\frac{1}{r} \partial_{r}+\frac{m^{2}}{r^{2}}-J\right] f=s E f \quad(r<b),
$$

where $f$ is the nonzero component of the spinor. The solution is oscillatory and peaked near the origin rather than at $b$. For $r>b$ the solution decays. This sharply contrasts with Dirac bound states, which are exponentially peaked at $b$. An energetics analysis for a Schrödinger electron with spin-orbit coupling in the presence of a skyrmion texture was carried out in Ref. [40].

\section{APPENDIX B: TIGHT-BINDING MODEL FOR NUMERICS}

Numerical calculations were performed using a tightbinding approximation to Eq. (2), with an additional term to avoid fermion doubling. On a square lattice of lattice constant $a$, we took

$$
\begin{aligned}
H_{e}+H_{e S}= & v_{F} \sum_{\mathbf{r}} \frac{i}{2 a} \hat{c}_{\mathbf{r}}^{\dagger}\left[\sigma^{y}\left(\hat{c}_{\mathbf{r}+\hat{\mathbf{x}}}-\hat{c}_{\mathbf{r}-\hat{\mathbf{x}}}\right)-\sigma^{x}\left(\hat{c}_{\mathbf{r}+\hat{\mathbf{y}}}-\hat{c}_{\mathbf{r}-\hat{\mathbf{y}}}\right)\right] \\
& -v_{F} \sum_{\mathbf{r}, \mathbf{e}=\hat{\mathbf{x}}, \hat{\mathbf{y}}} \frac{1}{2 a} \hat{c}_{\mathbf{r}}^{\dagger} \sigma^{z}\left(2 \hat{c}_{\mathbf{r}}-\hat{c}_{\mathbf{r}+\mathbf{e}}-\hat{c}_{\mathbf{r}-\mathbf{e}}\right) \\
& -J \sum_{\mathbf{r}} \hat{c}_{\mathbf{r}}^{\dagger} \vec{S}_{\mathbf{r}} \cdot \vec{\sigma} \hat{c}_{\mathbf{r}} .
\end{aligned}
$$

We set $a=v_{F}=1$ throughout. In Figs. 1 and 2 we solved the spectrum of $H_{e}+H_{e S}$ above on a $50 \times 50$ lattice in the presence of the skyrmion textures shown. We plotted the bound states and the momentum cutoff $k_{\mathrm{UV}}$ below which the skyrmions are favorable to the uniform state. In the interacting case the momentum cutoff is implemented by keeping states within a certain range determined by $k_{\mathrm{Uv}}$ around charge neutrality. A similar procedure was used in Figs. 4 and 3 although $y$-translation invariance was leveraged to use a $1000 \times 500$ lattice and no $k_{\mathrm{UV}}$ was used in Fig. 4.
[1] D. J. Thouless, M. Kohmoto, M. P. Nightingale, and M. den Nijs, Quantized Hall Conductance in a Two-Dimensional Periodic Potential, Phys. Rev. Lett. 49, 405 (1982).
[2] F. D. M. Haldane, Model for a Quantum Hall Effect without Landau Levels: Condensed-Matter Realization of the Parity Anomaly, Phys. Rev. Lett. 61, 2015 (1988). 
[3] C. L. Kane and E. J. Mele, $Z_{2}$ Topological Order and the Quantum Spin Hall Effect, Phys. Rev. Lett. 95, 146802 (2005).

[4] M. Z. Hasan and C. L. Kane, Colloquium: Topological insulators, Rev. Mod. Phys. 82, 3045 (2010).

[5] J. M. Kosterlitz and D. J. Thouless, Ordering, metastability and phase transitions in two-dimensional systems, J. Phys. C: Solid State Phys. 6, 1181 (1973).

[6] C. Castelnovo, R. Moessner, and S. L. Sondhi, Magnetic monopoles in spin ice, Nature (London) 451, 42 (2008).

[7] U. K. Rößler, A. N. Bogdanov, and C. Pfleiderer, Spontaneous skyrmion ground states in magnetic metals, Nature (London) 442, 797 (2006).

[8] S. Mühlbauer, B. Binz, F. Jonietz, C. Pfleiderer, A. Rosch, A. Neubauer, R. Georgii, and Böni, Skyrmion lattice in a chiral magnet, Science 323, 915 (2009).

[9] N. Nagaosa and Y. Tokura, Topological properties and dynamics of magnetic skyrmions, Nat. Nanotechnol. 8, 899 (2013).

[10] J. Liu and L. Balents, Anomalous Hall Effect and Topological Defects in Antiferromagnetic Weyl Semimetals: $\mathrm{Mn}_{3} \mathrm{Sn} / \mathrm{Ge}$, Phys. Rev. Lett. 119, 087202 (2017).

[11] H. Ochoa and Y. Tserkovnyak, Quantum skyrmionics, Int. J. Mod. Phys. B 33, 1930005 (2019).

[12] X. Z. Yu, N. Kanazawa, W. Z. Zhang, T. Nagai, T. Hara, K. Kimoto, Y. Matsui, Y. Onose, and Y. Tokura, Skyrmion flow near room temperature in an ultralow current density, Nat. Commun. 3, 988 (2012).

[13] S. Woo, K. Litzius, B. Krüger, M.-Y. Im, L. Caretta, K. Richter, M. Mann, A. Krone, R. M. Reeve, M. Weigand, P. Agrawal, I. Lemesh, M.-A. Mawass, P. Fischer, M. Kläui, and G. S. D. Beach, Observation of room-temperature magnetic skyrmions and their current-driven dynamics in ultrathin metallic ferromagnets, Nat. Mater. 15, 501 (2016).

[14] T. Lin, H. Liu, S. Poellath, Y. Zhang, B. Ji, N. Lei, J. J. Yun, L. Xi, D. Z. Yang, T. Xing, Z. L. Wang, L. Sun, Y. Z. Wu, L. F. Yin, W. B. Wang, J. Shen, J. Zweck, C. H. Back, Y. G. Zhang, and W. S. Zhao, Observation of room-temperature magnetic skyrmions in $\mathrm{Pt} / \mathrm{Co} / \mathrm{W}$ structures with a large spin-orbit coupling, Phys. Rev. B 98, 174425 (2018).

[15] W. Jiang, P. Upadhyaya, W. Zhang, G. Yu, M. B. Jungfleisch, F. Y. Fradin, J. E. Pearson, Y. Tserkovnyak, K. L. Wang, O. Heinonen, S. G. E. te Velthuis, and A. Hoffmann, Blowing magnetic skyrmion bubbles, Science 349, 283 (2015).

[16] F. Büttner, I. Lemesh, and G. S. D. Beach, Theory of isolated magnetic skyrmions: From fundamentals to room temperature applications, Sci. Rep. 8, 4464 (2018).

[17] A. Bogdanov and A. Hubert, The stability of vortex-like structures in uniaxial ferromagnets, J. Magn. Magn. Mater. 195, 182 (1999).

[18] A. Fert, N. Reyren, and V. Cros, Magnetic skyrmions: advances in physics and potential applications, Nat. Rev. Mater. 2, 17031 (2017).

[19] K. Nomura and N. Nagaosa, Electric charging of magnetic textures on the surface of a topological insulator, Phys. Rev. B 82, 161401(R) (2010).

[20] H. M. Hurst, D. K. Efimkin, J. Zang, and V. Galitski, Charged skyrmions on the surface of a topological insulator, Phys. Rev. B 91, 060401(R) (2015).

[21] K. Yasuda, R. Wakatsuki, T. Morimoto, R. Yoshimi, A. Tsukazaki, K. S. Takahashi, M. Ezawa, M. Kawasaki, N.
Nagaosa, and Y. Tokura, Geometric Hall effects in topological insulator heterostructures, Nat. Phys. 12, 555 (2016).

[22] M. M. Otrokov, I. I. Klimovskikh, H. Bentmann, D. Estyunin, A. Zeugner, Z. S. Aliev, S. Gaß, A. U. B. Wolter, A. V. Koroleva, A. M. Shikin, M. Blanco-Rey, M. Hoffmann, I. P. Rusinov, A. Y. Vyazovskaya, S. V. Eremeev, Y. M. Koroteev, V. M. Kuznetsov, F. Freyse, J. Sánchez-Barriga, I. R. Amiraslanov et al., Prediction and observation of an antiferromagnetic topological insulator, Nature (London) 576, 416 (2019).

[23] Y. Gong, J. Guo, J. Li, K. Zhu, M. Liao, X. Liu, Q. Zhang, L. Gu, L. Tang, X. Feng, D. Zhang, W. Li, C. Song, L. Wang, P. Yu, X. Chen, Y. Wang, H. Yao, W. Duan, Y. Xu et al., Experimental realization of an intrinsic magnetic topological insulator, Chin. Phys. Lett. 36, 076801 (2019).

[24] L. Fu and C. L. Kane, Topological insulators with inversion symmetry, Phys. Rev. B 76, 045302 (2007).

[25] S. Wimmer, J. Sánchez-Barriga, P. Küppers, A. Ney, E. Schierle, F. Freyse, O. Caha, J. Michalicka, M. Liebmann, D. Primetzhofer, M. Hoffmann, A. Ernst, M. M. Otrokov, G. Bihlmayer, E. Weschke, B. Lake, E. V. Chulkov, M. Morgenstern, G. Bauer, G. Springholz et al., Ferromagnetic $\mathrm{MnSb}_{2} \mathrm{Te}_{4}$ : A topological insulator with magnetic gap closing at high Curie temperatures of 45-50 K, arXiv:2011.07052 [condmat.mtrl-sci].

[26] C. Lei, S. Chen, and A. H. MacDonald, Magnetized topological insulator multilayers, Proc. Natl. Acad. Sci. USA 117, 27224 (2020).

[27] T. Moriya, Anisotropic superexchange interaction and weak ferromagnetism, Phys. Rev. 120, 91 (1960).

[28] I. Dzyaloshinskii, A thermodynamic theory of weak ferromagnetism of antiferromagnetics, J. Phys. Chem. Solids 4, 241 (1958).

[29] I. Dzyaloshinskii, Theory of helicoidal structures in antiferromagnets. I. Nonmetals, Sov. Phys. JETP 19, 960 (1963).

[30] A. Bogdanov and A. Hubert, Thermodynamically stable magnetic vortex states in magnetic crystals, J. Magn. Magn. Mater. 138, 255 (1994).

[31] X. Z. Yu, N. Kanazawa, Y. Onose, K. Kimoto, W. Z. Zhang, S. Ishiwata, Y. Matsui, and Y. Tokura, Near room-temperature formation of a skyrmion crystal in thin-films of the helimagnet FeGe, Nat. Mater. 10, 106 (2011).

[32] Y. Onose, Y. Okamura, S. Seki, S. Ishiwata, and Y. Tokura, Observation of Magnetic Excitations of Skyrmion Crystal in a Helimagnetic Insulator $\mathrm{Cu}_{2} \mathrm{OSeO}_{3}$, Phys. Rev. Lett. 109, 037603 (2012).

[33] V. M. Kuchkin, B. Barton-Singer, F. N. Rybakov, S. Blügel, B. J. Schroers, and N. S. Kiselev, Magnetic skyrmions, chiral kinks, and holomorphic functions, Phys. Rev. B 102, 144422 (2020).

[34] J. H. Han, J. Zang, Z. Yang, J.-H. Park, and N. Nagaosa, Skyrmion lattice in a two-dimensional chiral magnet, Phys. Rev. B 82, 094429 (2010).

[35] B.-J. Yang and N. Nagaosa, Skyrmion quantum numbers and quantized pumping in two-dimensional topological chiral magnets, Phys. Rev. B 84, 245123 (2011).

[36] F. Freimuth, R. Bamler, Y. Mokrousov, and A. Rosch, Phasespace Berry phases in chiral magnets: Dzyaloshinskii-Moriya interaction and the charge of skyrmions, Phys. Rev. B 88, 214409 (2013). 
[37] S. L. Sondhi, A. Karlhede, S. A. Kivelson, and E. H. Rezayi, Skyrmions and the crossover from the integer to fractional quantum Hall effect at small Zeeman energies, Phys. Rev. B 47, 16419 (1993).

[38] D.-H. Lee and C. L. Kane, Boson-Vortex-Skyrmion Duality, Spin-Singlet Fractional Quantum Hall Effect, and Spin-1/2 Anyon Superconductivity, Phys. Rev. Lett. 64, 1313 (1990).

[39] K. Moon, H. Mori, K. Yang, S. M. Girvin, A. H. MacDonald, L. Zheng, D. Yoshioka, and S.-C. Zhang, Spontaneous interlayer coherence in double-layer quantum Hall systems: Charged vortices and Kosterlitz-Thouless phase transitions, Phys. Rev. B 51, 5138 (1995).

[40] L. Brey, Magnetic skyrmionic polarons, Nano Lett. 17, 7358 (2017).

[41] S. E. Barrett, G. Dabbagh, L. N. Pfeiffer, K. W. West, and R. Tycko, Optically Pumped NMR Evidence for Finite-Size Skyrmions in GaAs Quantum Wells Near Landau Level Filling $v=1$, Phys. Rev. Lett. 74, 5112 (1995).

[42] H. Zhou, H. Polshyn, T. Taniguchi, K. Watanabe, and A. Young, Solids of quantum Hall skyrmions in graphene, Nat. Phys. 16, 154 (2020).

[43] R. Wakatsuki, M. Ezawa, and N. Nagaosa, Domain wall of a ferromagnet on a three-dimensional topological insulator, Sci. Rep. 5, 13638 (2015).

[44] F. Ye, G. H. Ding, H. Zhai, and Z. B. Su, Spin helix of magnetic impurities in two-dimensional helical metal, Europhys. Lett. 90, 47001 (2010).

[45] S. Zhang, F. Kronast, G. van der Laan, and T. Hesjedal, Realspace observation of skyrmionium in a ferromagnet-magnetic topological insulator heterostructure, Nano Lett. 18, 1057 (2018).

[46] S. Kim, K. Ueda, G. Go, P.-H. Jang, K.-J. Lee, A. Belabbes, and A. Manchon, Correlation of the Dzyaloshinskii-Moriya interaction with Heisenberg exchange and orbital asphericity, Nat. Commun. 9, 1648 (2018).

[47] S. Sorn, S. Divic, and A. Paramekanti, Tunable skyrmion crystals and topological quantum oscillations in magnetic metals, Phys. Rev. B 100, 174411 (2019).

[48] B. Ludbrook, G. Dubuis, A.-H. Puichaud, B. J. Ruck, and S. Granville, Nucleation and annihilation of skyrmions in $\mathrm{Mn}_{2} \mathrm{CoAl}$ observed through the topological Hall effect, Sci. Rep. 7, 13620 (2017).

[49] C. Liu, Y. Zang, W. Ruan, Y. Gong, K. He, X. Ma, Q.-K. Xue, and Y. Wang, Dimensional Crossover-Induced Topological Hall Effect in a Magnetic Topological Insulator, Phys. Rev. Lett. 119, 176809 (2017).

[50] J. Matsuno, N. Ogawa, K. Yasuda, F. Kagawa, W. Koshibae, N. Nagaosa, Y. Tokura, and M. Kawasaki, Interface-driven topological Hall effect in $\mathrm{SrRuO}_{3}-\mathrm{SrIrO}_{3}$ bilayer, Sci. Adv. 2, e1600304 (2016).

[51] L. Vistoli, W. Wang, A. Sander, Q. Zhu, B. Casals, R. Cichelero, A. Barthélémy, S. Fusil, G. Herranz, S. Valencia, R. Abrudan, E. Weschke, K. Nakazawa, H. Kohno, J. Santamaria, W. Wu, V. Garcia, and M. Bibes, Giant topological Hall effect in correlated oxide thin films. Nat. Phys. 15, 67 (2018).

[52] K. M. Fijalkowski, M. Hartl, M. Winnerlein, P. Mandal, S. Schreyeck, K. Brunner, C. Gould, and L. W. Molenkamp, Coexistence of Surface and Bulk Ferromagnetism Mimics Skyrmion Hall Effect in a Topological Insulator, Phys. Rev. X 10, 011012 (2020).

[53] S. Divic, H. Ling, T. Pereg-Barnea, and A. Paramekanti, Magnetic skyrmion crystal at a topological insulator surface, arXiv:2103.15841 [cond-mat.mes-hall].

[54] C.-K. Li, X.-P. Yao, and G. Chen, Twisted magnetic topological insulators, Phys. Rev. Research 3, 033156 (2021). 